\title{
Therapeutic Potential of Phthalimide Derivatives: A Review
}

\author{
José Guedes da Silva Júnior*1, Vanderlan Nogueira Holanda ${ }^{1}$, Daywison Silva Rodrigues \\ Gambôa1, Talyta Valéria Siqueira do Monte², Hallysson Douglas Andrade de Araújo, José \\ Adelson Alves do Nascimento Júnior ${ }^{1}$ Victor Felipe da Silva Araújo ${ }^{1}$, Maria Auxiliadora \\ Macedo Callôu ${ }^{1}$, Shalom Pôrto de Oliveira Assis ${ }^{3}$, Vera Lúcia Menezes Lima ${ }^{1}$ \\ ${ }^{1}$ Laboratório de Lipídeos e Biomoléculas e suas Aplicações em Doenças Prevalentes e Negligenciadas, Departamento de Bioquímica, Centro de Biociências, \\ Universidade Federal de Pernambuco, Brasil.
}

${ }^{2}$ Hospital Otávio de Freitas, Recife, Pernambuco, Brasil.

${ }^{3}$ Universidade Católica de Pernambuco, Brasil.

*Corresponding author: G SilvaJunior Jose, Laboratório de Lipídeos e Biomoléculas e suas Aplicações em Doenças Prevalentes e Negligenciadas, Departamento de Bioquímica, Centro de Biociências, Universidade Federal de Pernambuco, Brasil.

To Cite This Article: G SilvaJunior Jose. Therapeutic Potential of Phthalimide Derivatives: A Review. Am J Biomed Sci \& Res. 2019 - 3(4). AJBSR. MS.ID.000699. DOI: 10.34297/AJBSR.2019.03.000699

Received: June 18, 2019 | Published: June 27, 2019

\begin{abstract}
Phthalimides belong to the group of cyclic imides and have as common chemical characteristic two two carbonyls bound to the same nitrogen. Such compounds are obtained by various organic synthetic processes, generally using phthalic anhydride as the precursor. Because they present various biological activities such as antinoceptive, anti-inflammatory, antitumor, anti-convulsant, antimicrobial, among other actions, these molecules have been object of study in several research centers worldwide. This review article is intended to provide highly relevant information on the biological activities of these compounds and their mechanisms of systemic action that have already been elucidated. The articles used in these studies were obtained through the Internet [from Elsevier, NCBI and Science direct] in the period of July and August of 2018. Several compounds were identified acting in various pharmacological actions. The phthalimides and their derivatives studied in this study presented individual molecules, but also conjugated with other pharmacophoric groups, in order to improve their pharmacokinetic and pharmacodynamic aspects and thus generate better therapeutic responses. In spite of the studies with phthalimides, it is still necessary to investigate, mainly the mechanisms of action of these molecules, explaining all the interactions that they can make, so that new and more audacious studies can be carried out.
\end{abstract}

\section{Introduction}

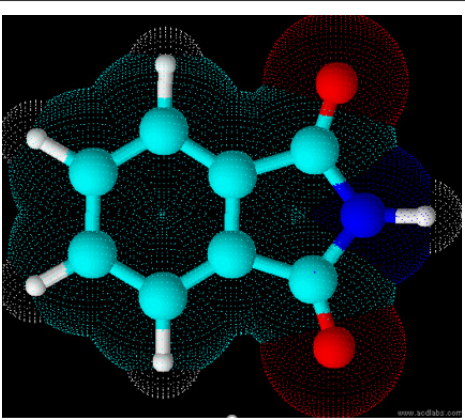

Figure 1: Model of the phthalimide molecule with the two carboxyls attached to the secondary amine. Molecule obtained in ChemSketch program 12.0, 2018

Pharmaceutical innovation has promoted great therapeutic advances against the most diverse diseases, be it infectious, metabolic or oncological [1]. These new therapeutic molecules have allowed a significant improvement in the quality of health worldwide. In the knowledge economy there is no productivity and no new pharmacological agents without a strong scientific and technological base, so encouraging the search for new pharmacophoric groups is fundamental for the progress of health systems [2]. Within the class 
of the cyclic imides where they are inserted, glutarimides, maleimides, succinimides and the naphtalimides, the phtalimides, a class of compounds with several biological actions already mentioned, stand out [3]. Like the other cyclic imides, phthalimides are characterized by a pair of carbonyls that are bound to the same nitrogen atom (Figure 1) [4]. These compounds have a number of similar characteristics such as hydrophobicity, which assures greater bioavailability and thus greater membrane diffusion capacity [5]
Phthalimides heterocyclic derivatives present several biological activities already described in the literature and this has attracted the attention of the scientific community [6]. Many phthalimide derivatives have been promising for the following biological activities: hypolipidemic [7-9], analgesic [10], anticonvulsant, anti-inflammatory [10,11], antiviral [12], antitubercular [9-13], and antimicrobials (Figure 2) [13-14].

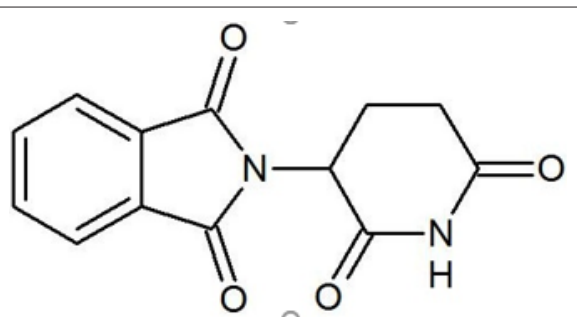

Figure 2: Chemical structure of Thalidomide, two amide rings and a single chiral center that form teratogenic and (R) teratogenic isomers with sedative effects. Molecule obtained in Chem Sketch program 12.0, 2018.

\section{Hypolipidemic activity}

Obesity is defined as the accumulation of fat in the organism in association with health risks due to its relationship with metabolic complications. This condition progresses when there is an imbalance between the calories consumed and the calories consumed, contributing also to this process: endocrinological disorders and sedentarisms, for example [15]. The major aggravating factor of diseases is the greater risk they cause for non-transmissible degenerative chronic diseases such as cardiovascular diseases, type 2 diabetes mellitus, musculoskeletal injuries such as osteoarthritis and some types of neoplasias [16]. Due to the serious risks that obesity can have on its patients, the search for therapies that can reduce high parameters such as hypercholesterolemia, hypetriglyceridemia and hyperglycemia is fundamental to avoid the installation of secondary complications that further aggravate the state of health of individuals. In this pharmacological aspect phthalimide derivatives have been presented as promising molecules in reducing high levels of cholesterol, triglycerides and serum glucose in animal models. In addition, some of these drugs have pleiotropic action, acting in a way to inhibit proinflammatory factors, which are released in obese individuals by adipose tissue [17].

The hypolipidemic action of phthalimide derivatives is one of the first activities described. Compounds containing 1-N-phthalimidobutane-3-one demonstrated significant hypocholesterolemic action; in another study, it was observed that, after 16 days of treatment [20 mg / kg / day] with 4.8-di-O-acetyl- 3-dideoxy- $\alpha$-D-erythro-hex-2-enopyranoside, there was a reduction of up to $25 \%$ in plasma cholesterol and triglycerides. After treatment of $20 \mathrm{mg} /$ $\mathrm{kg} /$ day mice with the phthalimideN- [4-chlorophenyl] phthalimide derivative, a $63-47 \%$ decrease in cholesterol and triglyceride levels, respectively, was observed. These studies demonstrate that compounds containing the phthalimide group are promising with hypolipidemic agents [7,18,19]. A new phthalimide derivative, LASSBio-1425, showed, among other activities, the ability to reduce serum levels of total cholesterol $[248.9 \pm 30.6 \times 519.2 \pm 17.4 \mathrm{mg}$ / dL], triglycerides 7, $3 \pm 7.8 \times 192.5 \pm 46.0 \mathrm{mg} / \mathrm{dL}]$, LDL [135.7 $\pm 31.2 \times 336.6 \pm 18.5 \mathrm{mg} / \mathrm{dL}]$ and increased HDL levels $[96.7 \pm$ $17.0 \times 25.4 \pm 3.8 \mathrm{mg} / \mathrm{dL}]$ in wistar rats fed a hypercholesterolemic diet. Other new halide cyclic imides with $\mathrm{N}$-phthalimide fraction presented significant hypolipidemic action in diabetic mice, with rates close to normal values, reinforcing the perspective of using phthalimide derivatives as hypolipidemic agents $[9,20]$.

\section{Analgesic activity}

Pain can be defined as a disagreeable unpleasant sensory and emotional experience that is related to a tissue or potential damage to the individual. The sensation of pain is responsive to several damages that the organism can be affected by virtue of physical traumas, inflammatory processes or of infectious or metabolic diseases of chronic character. The pain signal for the cerebral cortex follows the steps of transduction, conduction, transmission, perception and modulation of this signal. These mechanisms of nociception are composed of neuronal events that drive the noxious stimuli through nerve impulses to the central nervous system so that it perceives and generates a response [21]. The pharmacological search for agents that block the sensation of pain is strongly echoed in the scientific studies of medicinal chemistry, mainly due to some adverse effects that therapeutic agents used in clinical practice have, such as: dependence, hepatotoxicity, nephrotoxicity and negative effects on the gastrointestinal tract. As a result, new molecules with more potent analgesic action and with lower collateral effects are sought. Several studies have shown that analogues of phthalimide have an important antinoceptive effect [22].

Phthalimide derivatives also present analgesic activities with well established mechanisms already described in the literature (Figure 3). Seven new compounds containing the phthalimide group linked to methyl 1,2,4-oxadiazol-5-yl presented potent analgesic effect [ID50 $=2.2 \mathrm{mg} / \mathrm{kg} / \mathrm{ip}]$ through the acetic acid-induced abdominal writhing evaluation in mice. Recently, a group composed of 30 cyclic imides derived from phthalimide, was designed as selective inhibitors of COX-2 in order to obtain anti-inflammatory and analgesic activities. Among the derivatives, a potent inhibitor [IC50 $=0.18 \mu \mathrm{M}]$ and highly selective $[\mathrm{SI}=668]$ COX-2 $[10,23]$. 


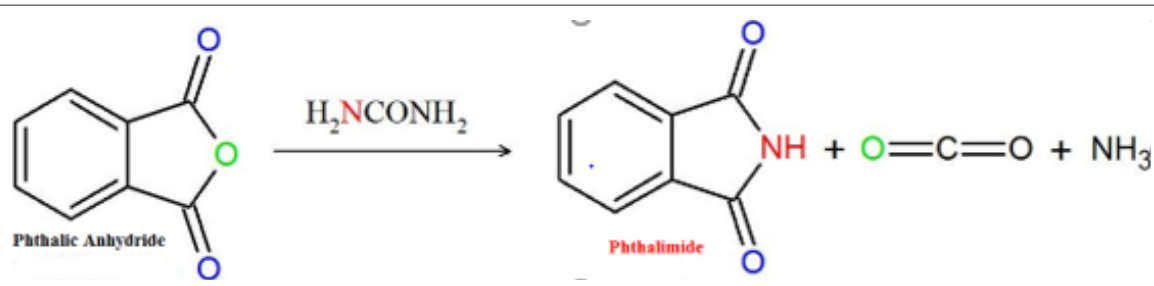

Figure 3: Chemical extraction of Phthalimides by Gabriel synthesis using urea in the reaction with phthalic anhydride provides a high yield Molecule obtained in ChemSketch program 12.0, 2018

\section{Anticonvulsant activity}

Some stress situations may lead to a state of electrophysiological disturbance in the central nervous system, resulting in a lack of synchrony of the neuronal activity, generating the state of convulsion. Situations such as hypoglycemia, physical trauma, meningitis and high febrile state can trigger a convulsive state. These convulsions can affect specific parts of the body, and can be of two main types: clonic when they cause involuntary movements on both sides of the body and tonic when there is sudden contraction of the skeletal muscles [24]. Therapy of seizures is varied and depends on the type and intensity of seizures that the patient presents. However, anticonvulsant drugs still present adverse reactions, such as Phenytoin that causes ataxia, vertigo, megaloblastic anemia, and even fetal malformations. Like Phenytoin, Carbamazepine also generates ataxia, in addition to fluid retention and leucopenia, which leads to complications secondary to the individual, which makes it possible to find compounds with an efficient action in blocking the disordered neuronal discharges and that present smaller adverse effects and toxic to the body [25].Anticonvulsant activity has also been reported after experiments with synthetic products containing phthalimide as the central component. Among the compounds described above, 2 phthalimide which showed protection against electrically induced seizures at doses of $30 \mathrm{mg} / \mathrm{kg}$ and $100 \mathrm{mg} / \mathrm{kg}$ and was shown to be more potent than phenytoin, a reference drug in the treatment of seizures [26].

Since 2000 with the hybridization of Thalidomide with Ameltolide, phthalimides have been studied as promising anticonvulsant agents, mainly because they have low side effects. A series of fluorophthalimides were tested to evaluate their effects on tonic and clonic convulsions in rats and the results showed that among the phthalimide derivatives with the aromatic double-ring compound, therefore more lipophilic, presented more satisfactory results than the control Phenytoin against clonic convulsions, not demonstrating the same effect on tonic manifestations. Seizure reactions were induced using pentylenetetrazole [27]. Another study using two series of phthalimides, one with phenyl ring and one with an $\mathrm{N}$-alkenyl, also inducing convulsion in rats with pentylenetetrazole observed that the alkenyl derivatives were more pharmacologically active against convulsion. This study also evaluated in silico the lipophilicity of these compounds using computational models and observed that there was no relation with higher lipophilicity of the molecules tested [28].

\section{Anti-inflammatory activity}

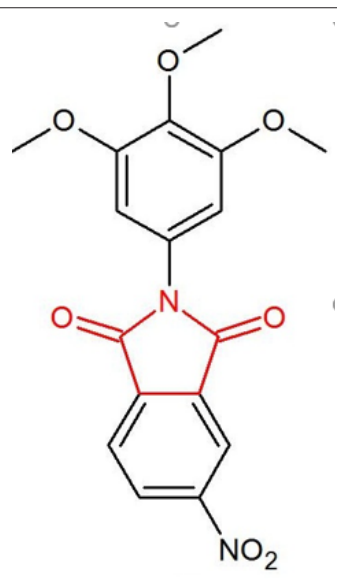

Figure 4: Chemical extraction of Phthalimides by Gabriel synthesis using urea in the reaction with phthalic anhydride provides a high yield. Molecule obtained in ChemSketch program 12.0, 2018

Another biological activity of phthalimides described in the literature is the anti-inflammatory action. A series of derivatives was synthesized to modulate the overproduction of TNF- $\alpha$, the primary mediator of the inflammatory response. It was observed that the phthalimide derivatives presented significant results when compared to TNF- $\alpha$ modulation at concentrations of 10 and $100 \mu \mathrm{M}$, surpassing thalidomide activity. In addition, the same derivatives were able to significantly inhibit [36\%] paw edema in mice treated with $10 \mathrm{mg} / \mathrm{kg}$ and decreased [45\%] carrageenan-induced hyperalgesia. In another study, where the 2-phthalimidethanol and 2-phthalimide derivatives were used, both inhibited paw edema when administered 1 hour before the application of carrageenan 
$[11,29]$. Another study using cyclic imides designed to have selective anti-inflammatory action against cyclooxygenase 2 [COX-2] was performed with 30 imidic compounds. Four of these compounds showed high anti-inflammatory action and ability to inhibit COX-2 with high selectivity index against this enzyme. The phthalimides tested showed a high structural reactivity with COX-2, similar to the COX-2 inhibitor Celecoxib. In this study the molecular docking analysis demonstrated that one of the phthalimidic derivatives has a binding mode with great specificity with COX-2 (Figure 4) [10].

Research using triazole-conjugated phthalimides demonstrated a high anti-inflammatory action of these compounds which were obtained through 1,3-dipolar cycloaddition cycle from $\mathrm{N}$ - [azidoalkyl] phthalimides and terminal alkynes. After the synthesis, purification and characterization of the compounds the anti-inflammatory potential of these cyclic imides in Swiss albino mice was evaluated by inducing inflammation with carrageenan through the plantar tissue of the right hind paw. All compounds showed anti-inflammatory activity, especially the 4-[4,6-di-0-acetyl-2,3-dideoxy- $\alpha$-D-erythro-hex-2-enopyranoside] -0-methyl-1 - [3-phthalimidopropyl] -1,2,3-triazole [3b] and 4- [N-Phthalimidomethyl] -1- [4-phthalimidobutyl] -1,2,3-triazole [5c] which showed $69 \%$ and $56 \%$ of anti-inflammatory activity [30].

One of the triggering mechanisms of the inflammatory process is the overproduction of nitric oxide [NO], which is also linked to the pathophysiology of other comorbidities involving infamation. Drugs that may inhibit the overproduction of nitric oxide are promising molecules that can act against several diseases of a more diverse nature. In light of this, a study using a series of phthalimide analogues tested these pharmacophoric groups against a lipopolysaccharide [LPS] -induced inflammatory process. In the tests cultures of RAW264.7 murine macrophages were used. In addition, an analysis of the structural relationship with biological activity showed that the free hydroxyls at the C-4 and C- 6 carbons along with the alkyl chain volume correlated positively with the anti-inflammatory potential. Phthalimide IIh showed an IC50 of $8.7 \mu \mathrm{g} /$ $\mathrm{ml}$. The researchers observed that the effects on NO reduction were not related to a cytotoxic activity of phthalimides, so the levels of nitric oxide synthase in the inducible isoform [iNOS] and mRNA expression were investigated [31]. For this investigation RAW264.7 cells were incubated for 12 hours with LPS and treated with compound IIh and others without phthalimide and subsequently Total RNA was isolated for Polymerase Chain Reaction [PCR] and the proteins analyzed by Western blot. The results demonstrated that cells that were not treated with the phthalimidic derivative IIh had a marked elevation of iNOS induced by LPS, unlike those treated with IIh which was capable of significantly suppressing the iNOS concentration and in a concentration dependent manner [31].

In addition, compound IIh promoted a reduction of proinflammatory cytokines such as Tumor Necrosis Factor-Alpha [TNF- $\alpha$ ], Interleukin $1 \beta$ [IL-1 $\beta]$ in the treated cells. The authors observed that the anti-inflammatory activity of compound IIh was linked to overexpression of the TLR-4 Tool-like receptor, which is activated by LPS and elicits a cascade of signals leading to the synthesis of pre-o inflammatory cytokines and mediators of inflammatory pro- cess such as nitric oxide. This TLR-4 signaling is divided into two ways: MyD88-dependent and MyD88-independent. The dependent pathway leads to the activation of the kappa nuclear factor protein complex B [NF-kB] which results in a greater expression of proinflammatory cytokines. The independent pathway leads to the expression of interferon- $\beta$. Acting on these signaling cascades, phthalimide IIh reduced the production of nitric oxide, TNF- $\alpha$, IL$1 \beta$ and iNOS, which credited it as a strong candidate for anti-inflammatory drug [31].

\section{Antimicrobial activity}

Despite the diverse metabolic activities of phthalimide derivatives, the antimicrobial assays deserve to be highlighted by the breadth of action of these compounds and their excellence in the fight against pathogenic microorganisms. Many infections by viruses, bacteria, protozoa, fungi and so on. drug cocktails, including potent drugs that bring the user several side effects, in addition to requiring long-term and multi-drug therapies [32]. The antiviral potential of new compounds, whether of synthetic or natural origin, has been of great interest throughout the development of science. The N-I-adamantyl-4-aminophthalimide derivative was prominent when it presented antiviral activity, with EC $50=4.78$ $\mu \mathrm{g} / \mathrm{mL}$ for the HIV virus. In the same study, the importance of the amino group on the phthalyl moiety in the biological activity investigated was emphasized, since other compounds with significant results also present the same chemical characteristic in common. A series of ftobuzone analogues, prepared from potassium phthalimide, showed significant inhibitory results against herpes simplex virus 1 and 2. The compounds with phthalimide moieties presented as potential antiviral agents, with IC $50=2.85 \mu \mathrm{g} / \mathrm{mL}$ for herpes simplex viruses 1 [33,34].

Other pyrimidine ring-containing phthalimide derivatives were also synthesized and tested against M. tuberculosis and showed significant values of Minimum Inhibitory Concentration [MIC] $=3.9 \mu \mathrm{g}$ / mL, cytotoxicity [CC50] $=312.5$, and selectivity index to macrophages [J774] of 80. Fluorinated phthalimide derivatives were also tested for efficacy in combating the tuberculous agent $\mathrm{M}$. tuberculossis H37Ra [ATCC 25177] and it was found that the fluorinated phthalimide hybrid molecules had similar anti-mycobacterial activity sulfonamide drugs. In another study, in which 12 phthalimide derivatives with fluorinated radicals were synthesized, the compounds showed potent antimicrobial activities, with a mean MIC of $1.8 \mu \mathrm{g} / \mathrm{mL}[13,35,36]$. The analogs obtained from the synthesis of $\mathrm{N}$ - [triethoxysilylpropyl] phthalimide by the transimidation reaction between phthalic anhydride and 3-aminopropyl triethoxysilane were tested for possible antimicrobial effect on strains of Staphylococcus aureus [ATCC 25923], Acinetobacter baumannii [ATCC 19606], Pseudomonas aeruginosa [ATCC 27853] and Escherichia coli [ATCC 25922]. The drugs tested were considered to be effective antimicrobials against all tested microorganisms, and the compounds with the phthalimidic structure showed a significantly higher result, and this effect can be attributed to the presence of phthalimides in their chemical composition [14].

The compounds N-vinylphthalimide and 8- [4- [phthalimid2-yl] butyloxy] quinoline, phthalimide derivatives obtained by 
modified condensation reactions, are described in the literature as good fungicidal agents. Synthetic analogues were tested against phytopathogens, Alternaria solani and Botrytis cinerea, with IC 50 values of $7.92 \mu \mathrm{g} / \mathrm{mL}$ and $10.85 \mu \mathrm{g} / \mathrm{mL}$ [37]. Other compounds containing the phtalimide group also showed excellent activity against the etiologic agent of malaria, Plasmodium falciparum. In general, in the study, the phthalimide group was introduced into [+] - dehydroabietylamine and from further reactions, twelve derivatives were obtained. Among the compounds obtained, 12-acetyl-N, N-phthaloylhydroabietylamine showed excellent activity against the protozoan with EC [antiplasmodic activity] $=0.086 \mu \mathrm{M}$ and SI [selectivity index] => 290, confirming that compounds with phthalimide grouping are promising in the treatment of intracellular protozoal infections [38].

\section{Antitumor activity}

Research with phthalimides with antitumor action has grown significantly, mainly because some types of cancer present resistance to medications already used, which reinforces the need to seek new antimitotic molecules. Blockage of tumor growth may oc- cur in one or more of the six phenotypes of the cancer cell that confer indiscriminate proliferative capacity. the disruption of the activity of cell signaling pathways for proliferation is one of the main therapeutic pathways used to curb tumor growth. Many phthalimides have been proposed as promising chemotherapeutic agents to block cell growth signaling pathways [39]. Like P13K / Akt / mTOR, a signaling pathway linked to cell growth, differentiation and differentiation, and when altered, are operative for initiation and tumor development. A study using glycoconjugated phthalimides demonstrated that these compounds showed antitumor activity by being able to inhibit the enzymes of the P13K pathway by means of proteolysis. In addition, the compounds regulated the phosphorylation of the Akt, S6K and GSK-3 $\beta$ pathway in cells of the HepG2 hepatic lineage. One of the study's phthalimide molecules was even more promising by inhibiting cell proliferation and inducing an autophagy process in a process that includes the formation of autophagosomes, fusion with lysosomes, and degradation of cell structures. This process was evidenced by the conversion of autophagy marker protein LC3BI and LC3BII detected by western blot (Figure 5) [40].

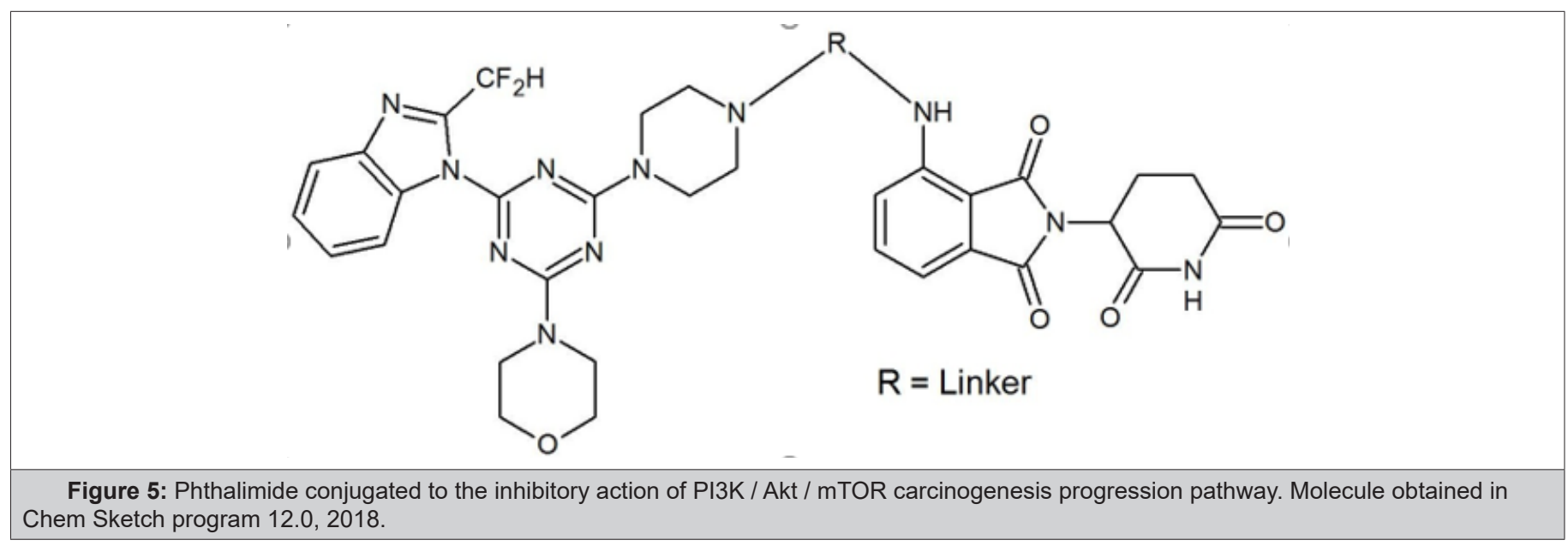

A number of chemical derivatives of thalidomide and phthalimide esters were synthesized and purified for evaluation of their antitumor potential against HepG2 cell lines of liver cancer and MCF-7 mammary neoplasia. One of the phthalimide esters demonstrated high inhibition of MCF-7 lineage growth, which was not observed by the other molecules. None of the compounds were effective in inhibiting the hepatic lineage. However, three phthalimidic derivatives elicited an immunosuppressive effect and six an immunostimulatory effectOf the compounds tested two Thalidomide derivatives and a phthalimide ester showed a high potential to inhibit vascular endothelial growth factor [VEGF] that in this study was used a recombinant form [VEGFR] and analyzed by sandwich Elisa [41]. Phthalimide derivatives have also been proposed as anticancer therapeutic agents in combination with chemotherapeutics already used in antimitotic therapies. A study with Oprozomib [OPZ] a protease inhibitor and Pomalidomide [Pom] a phthalimidic derivative of thalidomide has shown that phthalimide in a study of antiangiogenic action on choroalanoid membrane against the development of multiple myolaemic.
This study showed that Oprozomib was able to significantly reduce neoangiogenesis and expression of genes related to endothelial protein synthesis. The study then found that when Oprozomib was combined with dexamethasone [Dex] or with Pomalidomide the effects were even more significant when compared with monotherapy. There was even more therapeutic significance when the three molecules [OPZ + Pom + Dex] were used, demonstrating that the pharmacological combinations may contribute to a better therapeutic efficacy against cancer. In addition, this study using animal model allows to investigate other forms of phthalimide derivatives, such as the tumor microenvironment, the transition of the mensenchymal epithelium and mainly the angiogenesis process, which are not analyzed in cell culture analyzes [42].

\section{Toxicity}

Studies using phthalimides and their derivatives have shown low toxic effects at the acute level of these molecules. In a study with arylphthalimides and its hypolipidemic and anti-inflammatory activity, a therapeutic safety test was performed to determine its 
acute toxicity [LD50]. The test demonstrated that the therapeutic dose for the biological activities tested [ $250 \mathrm{mg} / \mathrm{kg}$ ] was safe, since even at the highest tested dose of $2 \mathrm{~g} / \mathrm{kg}$ there was no lethality and neither did the animals show signs of adverse reactions such as behavioral changes such as twitching, breathing breathlessness, palpitations, and decreased respiratory rate (Figure 6) [43].

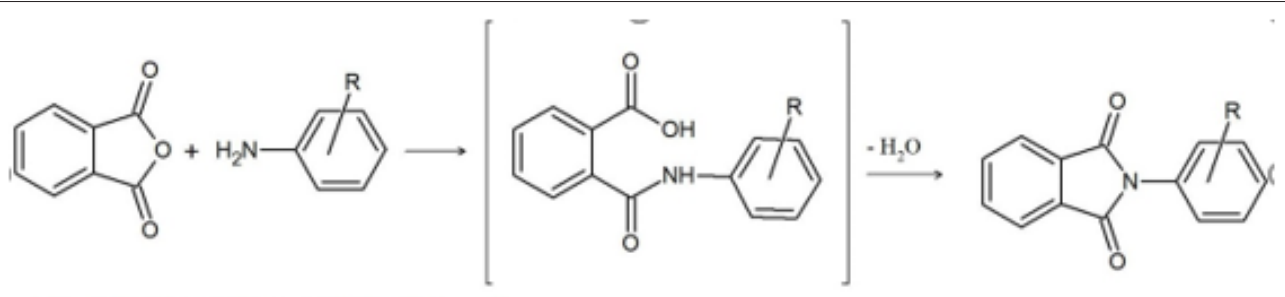

$\mathrm{R}=\mathrm{H}, \mathrm{O}-\mathrm{N}_{2} \mathrm{O}, m-\mathrm{N}_{2} \mathrm{O}, \mathrm{p}-\mathrm{N}_{2} \mathrm{O}, \mathrm{p}-\mathrm{Br}, \mathrm{p}-\mathrm{CONH} \mathrm{N}_{2}, \mathrm{p}-\mathrm{MeS}$ ou $\mathrm{p}-\mathrm{Cl}$

Figure 6: Synthetic pathways to obtain arylphthalimides with anti-inflammatory action. Molecule obtained in ChemSketch program 12.0, 2018.

\section{Conclusion}

Despite the many advances in modern medicine, many diseases of a diversified nature still cause various health complications and deaths. Pharmacological treatments are one of the main alternative to seek resolution or placate symptoms of diseases. In this scenario, the compounds originated from organic synthesis are highlighted because they are quickly obtained molecules and do not face problems of sustainability and seasonality as the compounds extracted from bioprospecting. Among the various classes of organic synthesis molecules, phthalimides are distinguished by their extremely pharmacological effects and often pleiotropic, acting on more than one front in the same organism. The actions of these compounds are from antimicrobial agents, mainly against fungi, bacteria and parasites, to effects on the metabolism of macromolecules and regulation of the processes of pain and inflammation. This great variability of pharmacological actions along with the constant possibility of improvement and conjugation of the phthalimide molecules so that they become increasingly active against the various insults and less toxic, places them in a prominent place in the scenario of medicinal chemistry, so that more and more this important class of cyclic imides is elucidated, until it materializes in effective therapeutic tools against diverse comorbidities.

\section{References}

1. Verweij J, Hendriks HR, Zwierzina H (2019) Innovation in Oncology Clinical Trial design. Cancer Treat Rev 74: 15-20.

2. Daniel GW, Cazé A, Romine MH, Audibert C, Leff JS, et al. (2015) Improving Pharmaceutical Innovation by Building a more comprehensive Database on Drug Development and use. Health Aff (Millwood) 34(2): 319-327.

3. Yuan YC, Bruneau C, Dorcet V, Roisnel T, Gramage-Doria R, et al. (2019) Ru-Catalyzed Selective C-H Bond Hydroxylation of Cyclic lmides. J Org Chem 84(4): 1898-1907.

4. Lian F, Sun C, Xu K, Zeng C 2019) Electrochemical Dehydrogenative Imidation of N-Methyl-Substituted Benzylamines with Phthalimides for the Direct Synthesis of Phthalimide-Protected gem-Diamines. Org Lett 21(1): 156-159.

5. Georgiev A, Stoilova A, Dimov D, Yordanov D, Zhivkov I, et al. (2019) Synthesis and photochromic properties of some N-phthalimide azoazomethine dyes. A DFT quantum mechanical calculations on imineenamine tautomerism and trans-cis photoisomerization. Spectrochim Acta A Mol Biomol Spectrosc 210: 230-244.
6. Winter GE, Buckley DL, Paulk J, Roberts JM, Souza A, et al. (2015) Selective Target Protein Degradation via Phthalimide Conjugation. Science. 348(6241): 1376-1381.

7. Chapman Jr JM, Cocolas GH, Hall IH, (1983) Hypolipidemic activity of phthalimide derivatives IV: Further chemical modification and investigation of the hypolipidemic activity of $\mathrm{N}$-substituted imides. J Pharm Sci 72(11): 1344-1347.

8. Murthy A, Wyrick S, Hall, IH (1985) Hypolipidemic activity of indan-1, 3-dione derivatives in rodents. J Med Chem 28 (11): 1591-1596.

9. Abdel-Aziz AAM, El-Azab AS, Attiade SM, Al-ObaidaMohamed AM, AlOmara MA, et al. (2011) Synthesis and biological evaluation of some novel cyclic-imides as hypoglycaemic, anti-hyperlipidemic agents. Eur J Med Chem 46 (9): 4324-4329.

10. Alanazi AM, El-Azab AS, Al-Suwaidan IA, ElTahir KEH, Asiri YA, et al (2015) Structure-based design of phthalimide derivatives as potential cyclooxygenase-2 (COX-2) inhibitors: anti-inflammatory and analgesic activities. Eur J Med Chem 6(92):115-123.

11. Casal JJ, Bollini M, Lombardo ME, Bruno AM (2016) Thalidomide analogues: tumor necrosis factor-alpha inhibitors and their evaluation as anti-inflammatory agents. Eur J Pharm Sci 83:114-119.

12. Saari WS, Wai JS, Fisher TE, Craig MT, Hoffman JM, et al. (1992) Journal of Medicinal Chemistry. 35(21): 3792-3802.

13. Akgun H, Karamelekoglu I, Berk B, Kurnaz I, Sarıbıyı G, et al. (2012) Synthesis and antimycobacterial activity of some phthalimide derivatives. Bioorg Med Chem 20: 4149-4154.

14. Singh G, Saroa A, Girdhar S, Rani S, Sahoo S, et al. (2015) Synthesis, characterization, electronic absorption and antimicrobial studies of $\mathrm{N}$-(silatranylpropyl) phthalimide derived from phthalic anhydride. Inorganica Chimica Acta 427: 232-239.

15. Sharon (2017) MF Obesity: Risk factors, complications, and strategies for sustainable long-term weight management. J Am Assoc Nurse Pract 29(S1): S3-S14.

16. Martin-Rodriguez E, Guillen-Grima F, Martí A, Brugos-Larumbe A (2015) Comorbidity associated with obesity in alarge population: The APNA study. Obes Res Clin Pract 9(5): 435-447.

17. Mc Donnell DP, Park S, Goulet MT, Jasper J, Wardell SE, et al. (2014) Obesity, Cholesterol Metabolism and Breast Cancer Pathogenesis. Cancer Res 74(18): 4976-4982.

18. Srivastava RM, Oliveira FJ, da Silva LP, de Freitas Filho JR, Oliveira SP, et al. (2001) Synthesis and hypolipidemic activity of N-phthalimidomethyl tetra-0-acyl- $\alpha$-d-mannopyranosides. Carbohydr Res 332(3): 335-340.

19. Sena VLM, Srivastava MR, Silvaa SO, Lima VLM (2003) Synthesis and hypolipidemic activity of $\mathrm{N}$-substituted phthalimides. Part V. Farmaco 58(12): 1283-1288. 
20. Fumian MM, da Motta NA, Maia R, Fraga CA, Barreiro EJ, et al (2016) LASSBio-1425, an analog of thalidomide, decreases triglyceride and increases HDL cholesterol levels by inhibition of TNF- $\alpha$ production. Int J Cardiol 202: 497-499.

21. Vanderah TW 2007) Pathophysiology of Pain. The Medical Clinics of North America. 91(1): 1-12.

22. Silva GF, Anjos MF, Wunsch RL, Ferreira LFGR, Stiz DS, et al. (2017) Antihypersensitivity effects of the phthalimide derivative N-(4methylphenyl)4-methylphthalimide in different pain models in mice. Biomedicine \& Pharmacotherapy 96: 503-512.

23. Antunes R, Batista H, Srivastava RM, Thomas G, Araujo CC, et al. (1998) New phthalimide derivatives with potent analgesic activity: II. Bioorg Med Chem Lett 8(21): 3071-3076.

24. Asadi-Pooya AA (2017) Psychogenic nonepileptic seizures: a concise review. Neurol Sci 38(6): 935-940.

25. Gierbolini JR, Giarratano M, Benbadis SR (2016) Carbamazepine-related antiepileptic drugs for the treatment of epilepsy - a comparative review. Expert Opinion on Pharmacotherapy 17(7): 885-898.

26. Kamiński K, Obniska J, Wiklik B, Atamanyuk D (2011) Synthesis and anticonvulsant properties of new acetamide derivatives of phthalimide, and its saturated cyclohexane and norbornene analogs. Eur J Med Chem 46(9): 4634-4641.

27. Iman M, Fakhari S, Jahanpanah M, Naderi N, Davood A, et al. (2018) Design and Synthesis of 4-flurophthalimides as potential anticonvulsant agentes. Iranian Journal of Pharmaceutical Research 17(3): 896-905.

28. Wicek M, Kiec-Kononowicz K (2006) Synthesis and Anticonvulsant Evaluation of Some N-Substituted Phthalimides. Acta Pol Pharm 66(3): 249-257.

29. Coêlho LCD, Cardoso MVO, Moreira DRM, Gomes PATM, Cavalcant SMT, et al. (2014) Novel phthalimide derivatives with TNF- $\alpha$ and IL-1 $\beta$ expression inhibitory and apoptotic inducing properties. Med Chem Comm 5(6): 758-765.

30. Assis SPO, Silva MT, Oliveira RN, Lima VL (2012) Synthesis and AntiInflammatory Activity of New Alkyl-Substituted Phthalimide 1H-1,2,3Triazole Derivatives. Scientific World Journal 5: 1-7.

31. Bach DH, Liu JY, Kim WK, Hong JY, Park SH, et al. (2017) Synthesis and biological activity of new phthalimides as potential anti-inflammatory agents. Bioorg Med Chem 25(13): 3396-3405.

32. Bentrup ZKH, Russell DG (2001) Mycobacterial persistence: adaptation to a changing environment. Trends Microbiol 9 (12): 597-605.
33. Van Derpoorten K, Balzarini J, De Clercq E, Poupaert JH (1997) Anti-HIV activity of N-1-adamantyl-4-aminophthalimide. Biomed Pharmacother 51(10): 464-468.

34. Yang YJ, Zhao JH, Pan XD, Zhang PC, (2010) Chemical and Pharmaceutical Bulletin. 58(2): 208-211.

35. Santos JL, Yamasaki PR, Chin CM, Takashi CH, Pavan FR, et al. (2009) Synthesis and in vitro anti Mycobacterium tuberculosis activity of a series of phthalimide derivatives. Bioorg Med Chem 17 (11): 3795-3799.

36. Elumalai K, Ali MA, Elumalai M, Eluri K, Srinivasan S, et al. (2013) characterization and biological evaluation of acetazolamide, cycloserine and isoniazid condensed some novel phthalimide derivatives. International Journal of Chemical and Analytical Science 4 (2): 57-61.

37. Pan L, Li X, Gong C, Jin H, Qin B (2016) Synthesis of N-substituted phthalimides and their antifungal activity against Alternaria solani and Botrytis cinerea. Microb Pathog 95: 186-192.

38. González MA, Clark J, Connelly M, Rivas F (2014) Antimalarial activity of abietane ferruginol analogues possessing a phthalimide group. Bioorg Med Chem Lett 24(22): 5234-5237.

39. Belluti S, Orteca G, Semeghini V, Rigillo G, Parenti F, et al. (2019) Potent Anti-Cancer Properties of Phthalimide-Based Curcumin Derivatives on Prostate Tumor Cells. Int J Mol Sci 20 (1): 28.

40. Li W, Gao C, Zhao L, Yuan Z, Chen Y, et al. (2018) Phthalimide conjugations for the degradation of oncogenic PI3K. European Journal of Medicinal Chemistry. 2018; 10 (151): 237-247.

41.Zahran MA, Abdin YG, Osman AM, Gamal-Eldeen AM, Talaat RM, et al. (2014) Synthesis and Evaluation of Thalidomide and Phthalimide Esters as Antitumor Agents. Arch Pharm (Weinheim) 347(9): 642-649.

42. Sanchez E, Li M, Wang CS, Tang G, Gillespie A, et al. (2017) Antiangiogenic and anti-multiple myeloma effects of oprozomib (OPZ) alone and in combination with pomalidomide (Pom) and/or dexamethasone (Dex). Leuk Res 57: 45-54.

43. Assis SPO, Araújo TG, Sena VLM, Catanho MTJA, Ramos MN, et al. (2014) Synthesis, hypolipidemic, and anti-inflammatory activities of arylphthalimides. Medicinal Chemistry Research 23(2): 708-716. 